\title{
Geothermal power based ULTDH for cooling and heating purposes
}

\author{
Bartłomiej Ciapała ${ }^{1, *}$, and Mirosław Janowski ${ }^{1}$ \\ ${ }^{1}$ AGH University of Science and Technology, Faculty of Geology, Geophysics and Environmental \\ Protection, Department of Fossil Fuels
}

\begin{abstract}
Ultra-low temperature district heating systems facilitate use of waste and renewable heat sources. The article presents a possible scheme of operation and optimisation of small ultra-low temperature district heating system consisting of waste heat source, a number of heated individual dwellings and borehole thermal energy storage plant. Optimisation performed for typical meteorological year for Kraków indicate significant potential of decreasing energy amount discharged to the environment and total length of borehole heat exchangers, compared to individual heat/cold production from low-temperature geothermal resources. Meanwhile, satisfied is a set of constrains providing borehole thermal energy storage sustainability and fulfilling entire heating and cooling demands.
\end{abstract}

\section{Introduction}

Sufficient heating and cooling of buildings and industrial processes becomes one of the most crucial challenges in their proper design and realisation. Even more difficult is to meet requirements using environmentally friendly systems while keeping operation economically competitive. Especially, that the area and resources are usually limited. Common solution is an individual supplying in heat and cooling. It guarantees flexibility and potentially gives independency from external suppliers (which usually is illusive or no use is made of it). Simultaneously, such a solution entails entire variety of efforts including maintenance, optimal management, risk mitigation and other. Considering narrow specialisations of companies and regarding desire of comfortable and effortless maintaining internal temperature of flats and detached houses, many owners are likely to connect their buildings to the district heating. Therefore, some efforts are made to ease using district heating network not only for heating, but also for cooling [1]. Also, heat pumps in district heating gain in importance [2]. Among solutions prospective in this context, especially placed in not very densely built areas is ultra-low-temperature district heating (ULTDH) [3].

ULTDH is system, in which supply temperature is too low to prepare sanitary hot water (SHW) without an additional heat source [4]. Thus, it has potential to be both heat source and heat sink, as temperature in the pipeline may be low enough for heat discharge from many appliances [4]. Simultaneously, decreased temperature does not need to increase pipe's diameter compared to typical medium-temperature $\mathrm{DH}$, as heat pumps operation may result

* Corresponding author: bciapala@agh.edu.pl 
in similar temperature differences between upstream and downstream nodes. It is important to notice, that energy loss in the pipeline may be decreased in comparison with mediumtemperature $\mathrm{DH}$ and heat boosters may be used to meet end user's requirements [5-7].

The aim of this publication is to perform test and optimisation of ULTDH operating in conditions of Kraków city in Poland and compare results with stand-alone installations covering the same heating and cooling demands.

\section{Problem description}

The present article is to propose ultra-low-temperature district heating system used for cooling small industrial object, space heating and sanitary hot water preparation for citizens (this solution is already described and known from real-life conditions [4]) and energy gaining/storage from borehole heat exchangers (BHE), which constitute borehole thermal energy storage (BTES) (see Fig. 1.). The energy is recovered for utilisation with heat pumps installed individually at dwellings. As mentioned buildings may be easily heated and cooled using their own systems of borehole heat exchangers, comparison with such individual solution will be shown.

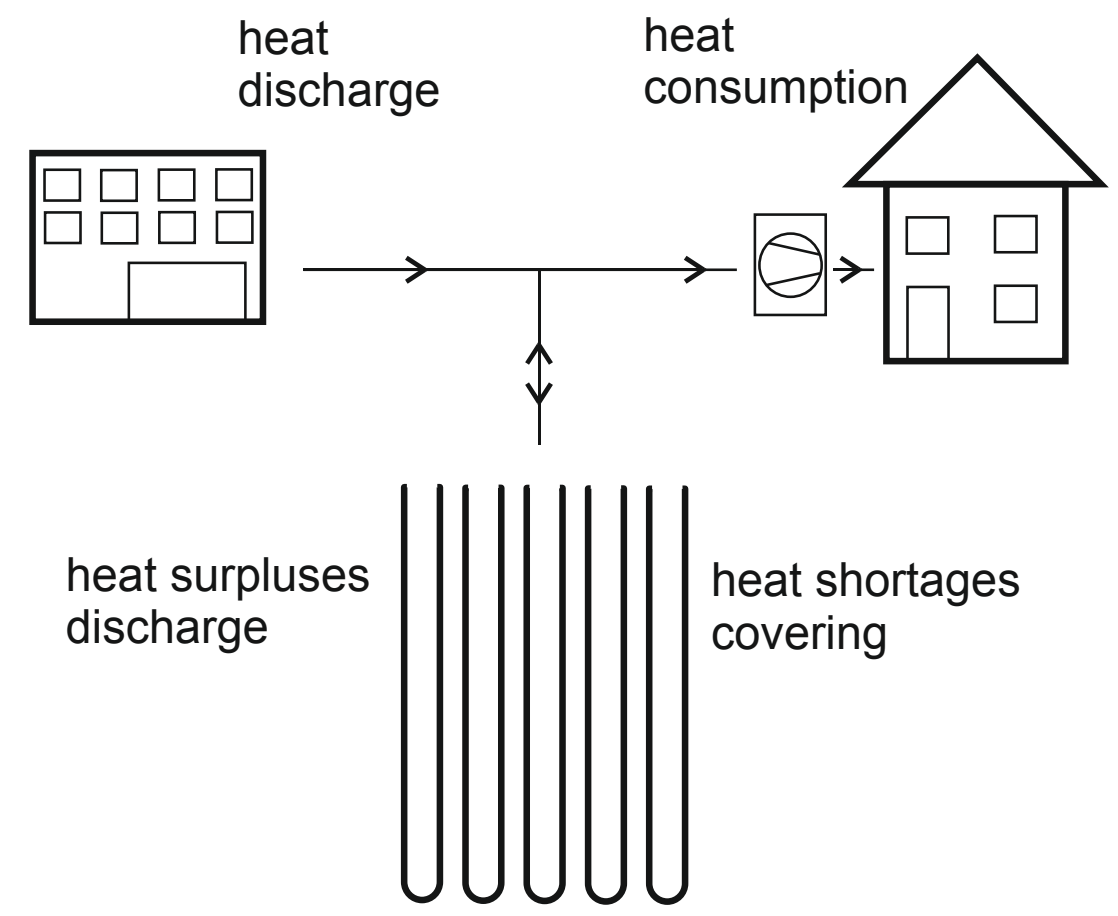

Fig. 1. Scheme of proposed system operation. Heat is discharged in a small industrial facility, while it is consumed in a dozen of individual dwellings/multi-storey building. Excess heat and its shortages are managed with borehole thermal energy storage.

Operation of such a system has been optimised in regard to the size of BTES and number of citizens whose living space heating and SHW preparation may be provided by proposed ULTDH system cooperating with small industrial facility with cooling demand (heating power discharged) varying from 64 to $210 \mathrm{~kW}$. Operation schedule may be observed on the graph Fig. 2. 


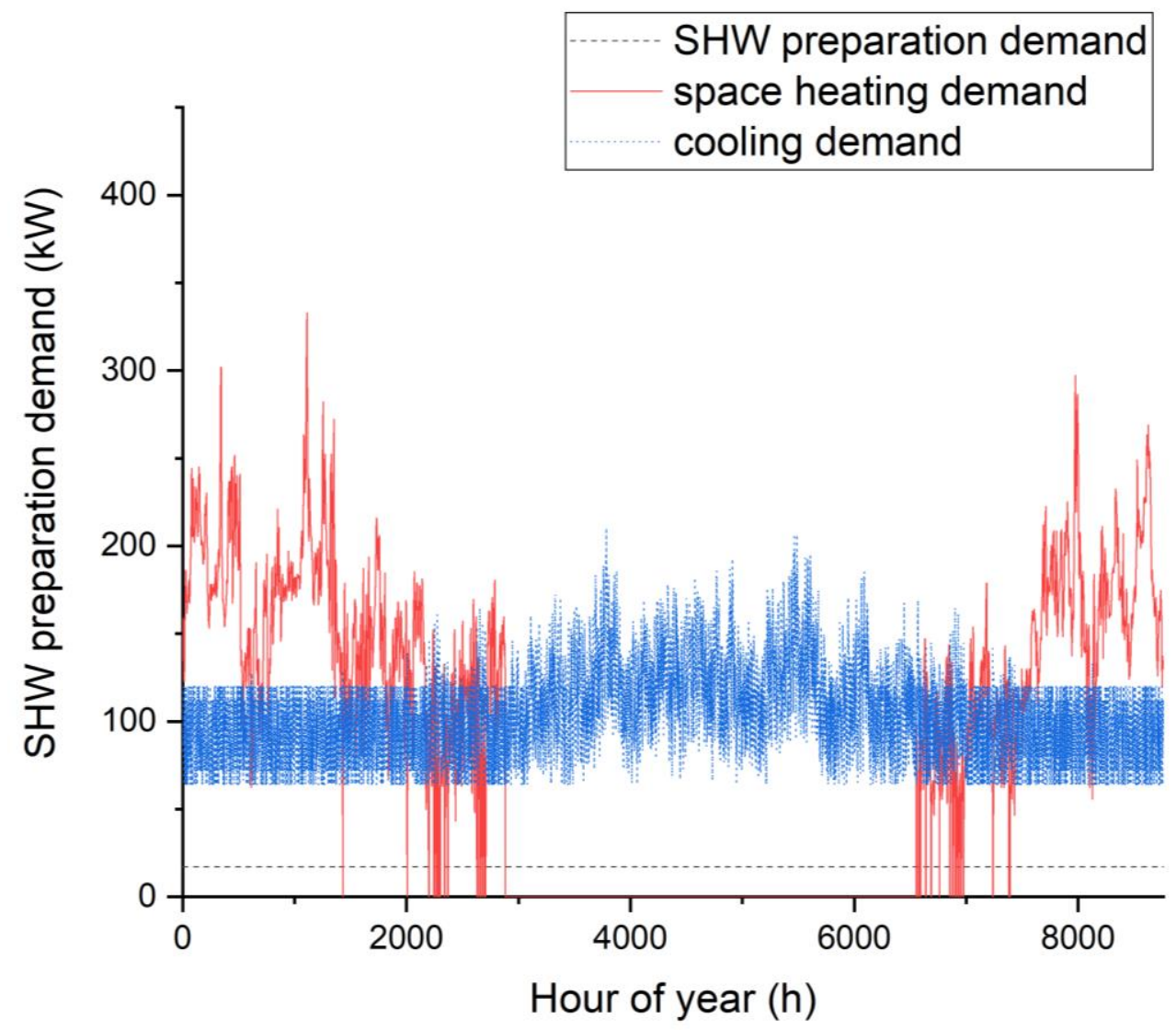

Fig. 2. Operation schedule of system receivers' demand for cold, heat and sanitary hot water preparation.

\section{Mathematical description of the problem}

Heat demand in hour $\mathrm{n}$ is described by the equation:

$$
E_{n}^{\text {heating }}=E^{S H W}+E^{\text {design }} \cdot \frac{T_{\text {designed }}^{\text {internal }} T_{n}^{\text {external }}}{T_{\text {designed }}^{\text {internal }}-T_{\text {designed }}^{\text {external }}}
$$

Where $E^{\text {design }} \cdot \frac{T_{\text {designed }}^{\text {internal }}-T_{n}^{\text {external }}}{T_{\text {designed }}^{\text {internal }}-T_{\text {designed }}^{\text {external }}}$ part is used to calculate energy demand for space heating in an hour n $\left(E_{n}^{\text {space heating }}\right)$.

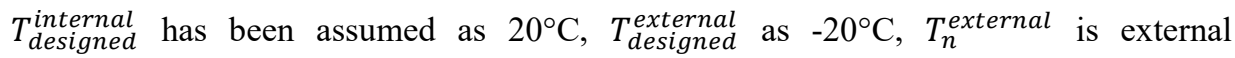
temperature as given in typical meteorological year (TMY) for Kraków Balice meteorological station. Design heat load $E^{\text {design }}$ equals $80 \mathrm{~W} / \mathrm{m}^{2}$ and total heated area is optimised and amount to $27.6 \mathrm{~m}^{2}$ of flat per capita, as mean for Poland (GUS). Space heating has been arbitrary disabled from May to September (no clear heating schedule is binding in Poland).

Heat energy required from ULTDH is calculated by the equation 


$$
E_{n}^{U L T D H \text { heating }}=\left(1-\frac{1}{C O P}\right) \cdot\left(E_{n}^{\text {space heating }}+E^{S H W}\right)
$$

Optimisation has been performed with objective function stated as

$$
Z=\min (H)
$$

Where $H$ is a total length of BHE.

With following constrains:

- Peak heating or cooling power cannot be higher than total BHE heat transfer rate potential

- $\quad$ BTES's energy content after an year of operation must be equal $+/-10 \%$ to its initial state of charge

- In any hour, BTES state of charge cannot be lower than 0

- In any hour, ULTDH must provide entire energy demand

An examined set of acceptable combinations is presented on Fig. 3.

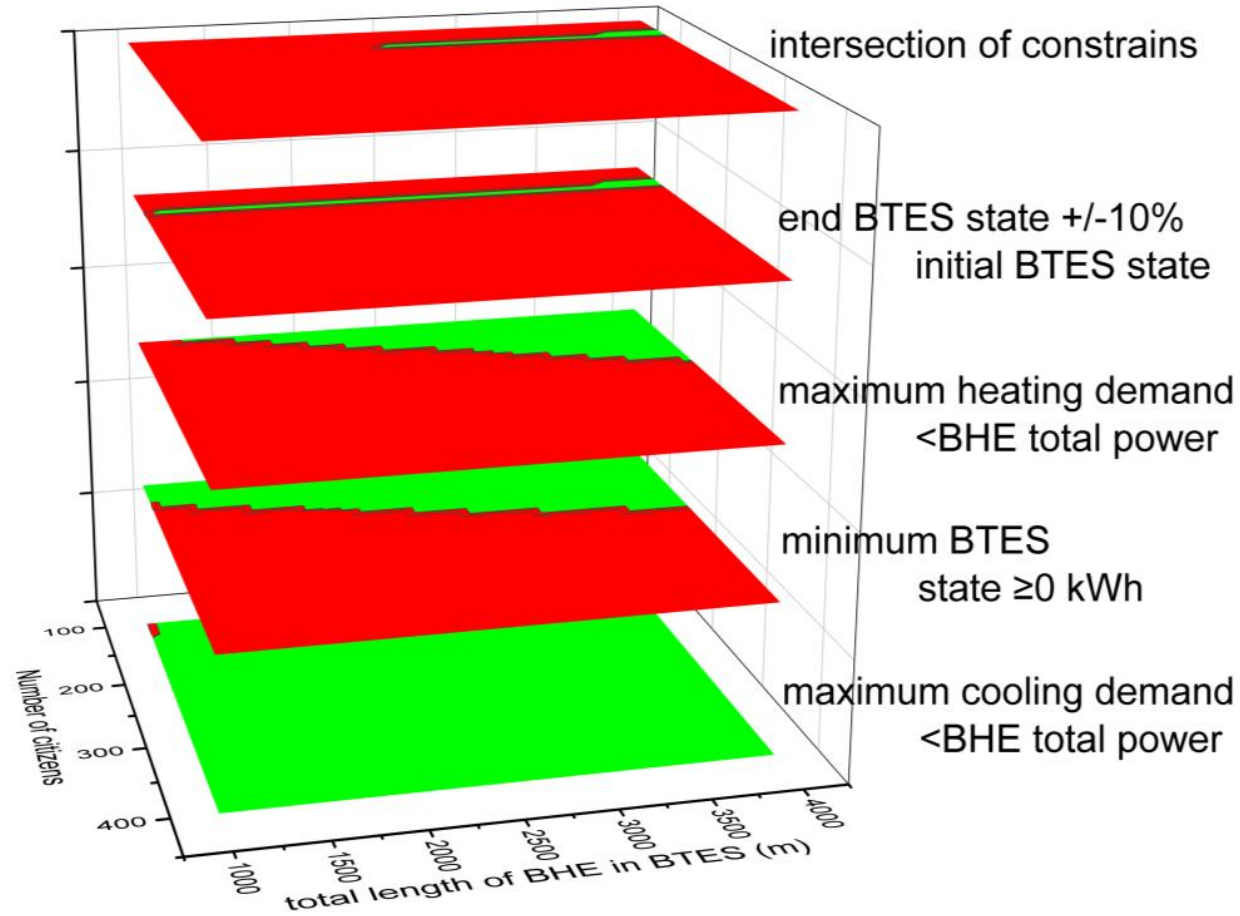

Fig. 3. Parameters' constrains. Green areas represent sets of parameters which do not violate constrains e.g. are acceptable. Otherwise - red parts.

The initial (in hour $\mathrm{n}=1$ ) BTES value has been set as $80 \mathrm{kWh}$ per meter of the borehole heat exchanger (BHE), which is moderate value for its energy content [8]. For each and every hour, energy provided by industrial facility is compared with energy demand decreased by $25 \%$, as heat pumps (HP) coefficient of performance (COP) equal to 4.0 has been assumed. Heat transfer rate of BHEs is assumed to be $40 \mathrm{~W} / \mathrm{m}$. If any excess occur, energy is stored in BTES of $40 \%$ efficiency [9]. Otherwise, lack of energy for space heating and domestic hot water preparation $\left(50 \mathrm{~kg}\right.$ per $24 \mathrm{~h}$ per person, temperature shift $50^{\circ} \mathrm{C}$, demand distributed steadily and equal in every hour) is covered by heat pump from BTES. For every hour $\mathrm{n}>1$, BTES energy content is described as: 


$$
E_{n}^{B T E S}=E_{n-1}^{B T E S}+0.4 \cdot E_{n}^{\text {cooling }}-\left(1-\frac{1}{C O P}\right) \cdot\left(E_{n}^{\text {space heating }}-E^{S H W}\right)
$$

Every hour, when

$$
\frac{E_{n}^{\text {cooling }}}{1-\frac{1}{C O P}}>E_{n}^{\text {space heating }}+E^{S H W}
$$

BTES is charged with excessing energy, otherwise - energy is extracted from BTES with $40 \%$ efficiency and extracted heat is calculated as:

$$
E_{n}^{B T E S \text { extracted }}=\left(1-\frac{1}{C O P}\right) \cdot\left(E_{n}^{\text {space heating }}+E^{S H W}\right)-\frac{E_{n}^{\text {cooling }}}{1-\frac{1}{C O P}}
$$

ULTDH transport heat loss has been neglected, as temperature difference between medium and ground would be small, supposedly less than $15^{\circ} \mathrm{C}$. Similarly, circulation pumps and heat capacity of DH medium is neglected.

\section{Results}

For given initial conditions, BTES consisting of about $2400 \mathrm{~m}$ of BHE is supposed to meet expectations. In its lowest energy content is $90 \mathrm{MWh}$, which is $47 \%$ of its initial state, while in its highest is $249 \mathrm{MWh}$ and $129.5 \%$ of BTES initial energy content. In total, for typical meteorological year BTES provided $430 \mathrm{MWh}$ of cooling and $180 \mathrm{MWh}$ for heating purposes. Simultaneously, receivers discharged $909 \mathrm{MWh}$ in cooling process and consumed $880 \mathrm{MWh}$ for space heating (note: $220 \mathrm{MWh}$ was produced from electricity in heat pump compressor, so total energy consumption was $880 \mathrm{MWh}$ ). Thus, significant amount of waste heat has been recovered and reused instead of being directly discharged into the environment, significantly reducing environmental burden. State of charge is presented in Fig. 4.

Over 8760 analysed hours, 780 BTES charging/discharging switching events occurred. The transient periods during which system is close to balance and BTES is hardly used are especially interesting, as represent system's highest efficiency. The final BTES state of charge indicates long-lasting system's durability and reliability, as its natural resource is not depleted. This leads to the conclusion that BHE might be even smaller, if its finite lifetime was assumed.

The simulation suggests that space heating and SHW preparation might be provided for 150 people. There is not a single hour in which heating and cooling demand would not be covered (what was one of the assumptions).

In comparison with individual sources of heat and cold for the same conditions, the BHE size is reduced by more than 5 times, bringing significant limitation on required space and capital costs. For typical conditions in Poland, about $60 \%$ of heat injected to BTES from cooling is dissipated in the environment. It is still not sufficient enough, yet significantly less than in case of traditional dry-coolers discharging heat into the atmosphere. Meanwhile, the heat discharged to ULTDH during space heating demand is consumed and significantly decreases conventional heat demand. Thus, heat is used efficiently, not susceptible for exergy decrease or heat storage inefficiency loss. For presented optimal conditions fulfilling all the constrains, share of heat discharged to ULTDH and directly used by heat pumps in overall heat demand is never lower than 50\% (Fig. 5). 


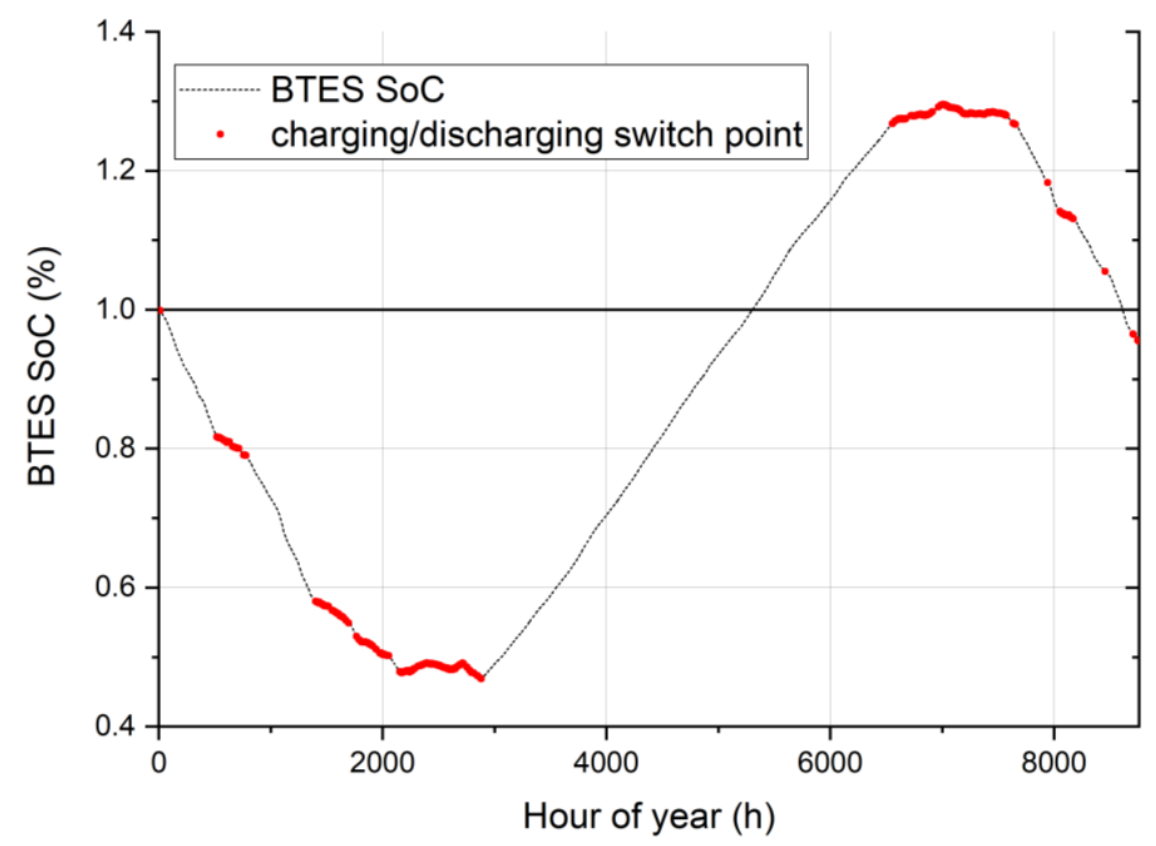

Fig. 4. State of charge of BTES. Red dots represent transient conditions, for which heat exchange in BTES occur in a very limited range, while entire ULTDH system's efficiency is highest.

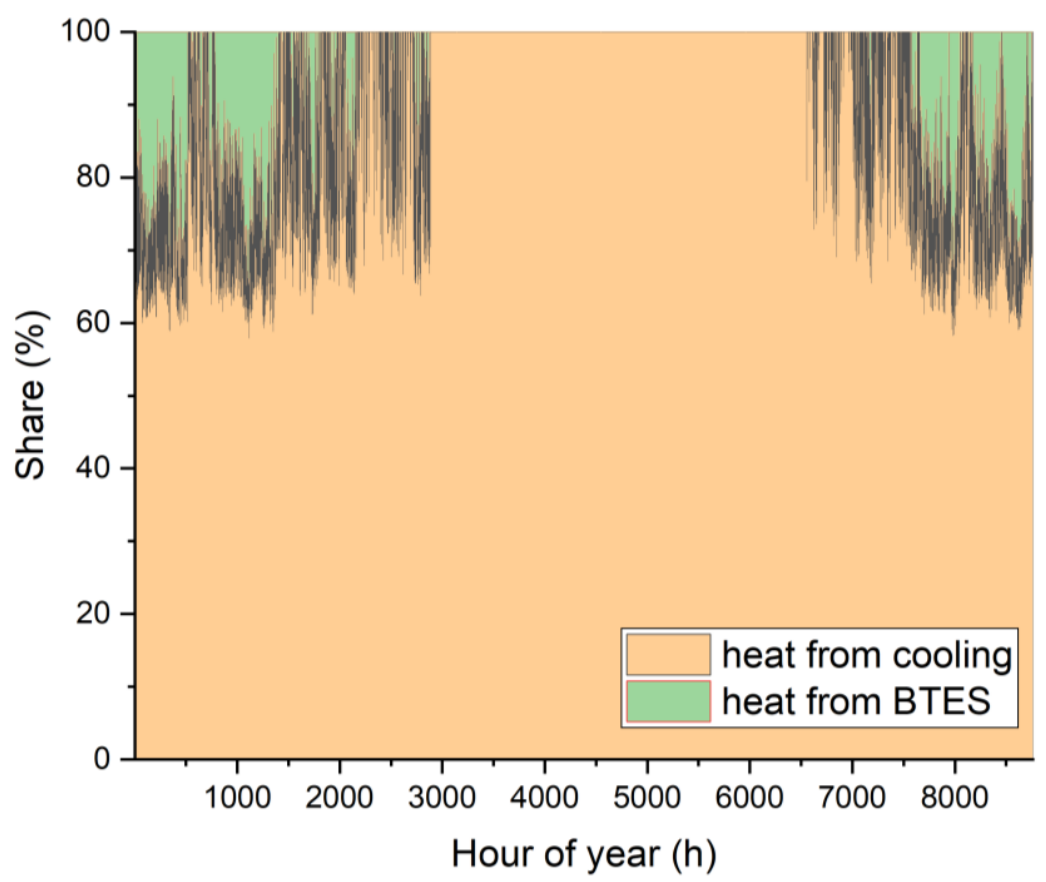

Fig. 5. Proportions of heat provided directly from cooling and from BTES. 


\section{Summary}

The optimisation and operation mode of the geothermal power based ULTDH for cooling and heating purposes has been presented. Obtained results indicate significant benefits derived from the ULTDH system operation connecting heat sources and heat sinks, which nominally are not dependent of each other. Low temperature of used medium brings benefits in limited heat losses and increased heat sink effectiveness. Total BHE length may be reduced 5 times compared to individual heat and cold sources while satisfying entire demands during the typical meteorological year. Simultaneously, less energy is dispersed in the environment, what has a potential in reducing negative effects like urban heat islands. Use of heat pumps at dwellings has potential to facilitate use of energy-efficient low temperature heating in houses (e.g. floor heating).

Presented results are valid for assumed situation during the typical meteorological year. Yet, the methodology is applicable in real-life considerations, where more complex and diversified data would be provided. For the future work, including DH system's heat capacity and performing sensitivity analyses for longer time periods is planned.

The paper has been prepared under the AGH-UST research grant No. 11.11.140.03.

\section{References}

1. B. Rezaie, M. A. Rosen, Appl. Energy, 93 (2012)

2. M. A. Sayegh, P. Jadwiszczak, B. P. Axcell, E. Niemierka, K. Bryś, H. Jouhara, Energy Build. 166 (2018)

3. B. Ciapała, J. Jurasz, M. Janowski, $10^{\text {th }}$ Conf. Interdiscip. Probl. Environ. Prot. Eng. EKO-DOK (2018)

4. O. Gudmundsson, M. Brand, J. E. Thorsen, The $14^{\text {th }}$ International Symposium on District Heating and Cooling (2014)

5. X. Yang, H. Li, S. Svendsen, Energy Convers Manag. 122 (2016)

6. T. Ommen, J. E. Thorsen, W. B. Markussen, B. Elmegaard, Energy, 137 (2017)

7. B. Ciapała, M. Janowski, J. Jurasz, Tech. Poszuk. Geol. Geoterm. Zrówn. Rozw. 2 (2018)

8. A. Casasso, R. Sethi, G. Pot, Energy, 106 (2016)

9. M. Lanahan, P. C. Tabares-Velasco, Energies 10, 6 (2017) 